\title{
A Catalog of Candidate High-redshift Blazars for GLAST
}

\author{
Tersi M. Arias \\ Office of Science, Science Undergraduate Laboratory Internship (SULI) \\ San Francisco State University \\ Stanford Linear Accelerator Center \\ Menlo Park, California
}

Aug 25, 2006

Prepared in partial fulfillment of the requirements of the Office of Science, U.S. Department of Energy's Science Undergraduate Laboratory Internship (SULI) Program under the direction of

J. Carson in the Kavli Institute of Particle Astrophysics and Cosmology at Stanford Linear Accelerator Center.

Participant:

Signature

Research Advisor:

\section{Signature}




\section{Table of Contents}

\section{Page}

Abstract

iii

Introduction

1

Methods

2

Results

Discussion and Conclusions

4

Acknowledgments

5

References

Tables

6

Figures

$6-8$ 


\begin{abstract}
A Catalog of Candidate High-redshift Blazars for GLAST. TERSI ARIAS (San Francisco State University, San Francisco, CA 94132) JENNIFER CARSON (Stanford Linear Accelerator Center, Menlo Park, CA 94025)
\end{abstract}

High-redshift blazars are promising candidates for detection by the Gamma-ray Large Area Space Telescope (GLAST). GLAST, expected to be launched in the Fall of 2007, is a highenergy gamma-ray observatory designed for making observations of celestial gamma-ray sources in the energy band extending from $10 \mathrm{MeV}$ to more than $200 \mathrm{GeV}$. It is estimated that GLAST will find several thousand blazars. The motivations for measuring the gamma-ray emission from distant blazars include the study of the high-energy emission processes occurring in these sources and an indirect measurement of the extragalactic background light. In anticipation of the launch of GLAST we have compiled a catalog of candidate high-redshift blazars. The criteria for sources chosen for the catalog were: high radio emission, high redshift, and a flat radio spectrum. A preliminary list of 307 radio sources brighter than $70 \mathrm{mJy}$ with a redshift $z \geq 2.5$ was acquired using data from the NASA Extragalactic Database. Flux measurements of each source were obtained at two or more radio frequencies from surveys and catalogs to calculate their radio spectral indices $\alpha$. The sources with a flat-radio spectrum $(\alpha \leq 0.5)$ were selected for the catalog, and the final catalog includes about 200 sources. 


\section{INTRODUCTION}

Blazars are active galactic nuclei (AGN) with relativistic plasma jets oriented close to the line of sight of the observer. These objects are intrinsically powerful variable radio sources and are the largest class of identified high-energy gamma-ray sources. Gamma-ray blazars are characterized by rapid flux variability and a high degree of linear polarization at visible wavelengths, with the measured rate of change limited by the statistics of the gamma-ray observations [1].

The study of high-energy emission processes in blazars has been difficult in the past because of the low number of gamma-ray blazars detected, especially at high redshifts. However, The Gamma-ray Large Area Space Telescope (GLAST), scheduled to launch in the Fall of 2007 will easily detect high-energy gamma-ray emission in the GeV range. GLAST will scan the entire sky every three hours and will provide long, evenly sampled, gamma-ray light curves for a large number of sources [2] including blazars. The much greater sensitivity of GLAST with respect to previous instruments should result in a large increase in the number of known highenergy sources, and it is estimated that GLAST will detect several thousand new gamma-ray blazars.

Spectral energy distribution (SED) is defined by power as a function of frequency $\left(v F_{v}\right.$ vs. $\left.v\right)$. The SED of blazars has a characteristic double peak signature, as shown in Figure 1, which suggests that synchrotron radiation and Compton scattering occurs in the jets. Electrons inside the jet are highly relativistic and are moved around by magnetic fields. The energy release ranges from relatively weak radio frequencies to powerful gamma-ray photons. High-energy gamma-ray emission is produced from inverse-Compton scattering of low-energy photons off relativistic electrons. The radio-frequency spectra and polarization properties of blazars are characteristics of synchrotron radiation from relativistic electrons having a power law distribution of electron energies [3]. Over small intervals the SED is characterized by a powerlaw distribution $F_{v} \propto v^{-\alpha}$, where $\alpha$ is the spectral index. A spectral index of $\alpha \leq 0.5$ is considered a flat radio spectrum and is characteristic of blazars. 
The study of absorption features imprinted on the gamma-ray spectra of distant blazars provides information about the extragalactic background light (EBL). The diffuse EBL consists of the sum of the starlight emitted by galaxies through the history of the universe. EBL causes attenuation of gamma-ray photons via pair production when they travel through space. Highredshift blazars should be significantly affected by EBL absorption and the further away they are, the more absorbed the gamma-ray spectrum will be.

This project focuses on gathering information from compact radio sources with high redshifts $(z \geq 2.5)$ and flat spectra $(\alpha \leq 0.5)$ from databases such as the Nasa Extragalactic Database (NED) and surveys such as the combined National Radio Astronomy Observatory (NRAO) and the Very Large Array (VLA) Sky Survey (NVSS). The goal is to build a catalog of

sources that are promising candidates for detection by GLAST. The GLAST measurements of these sources could provide new and extensive information about the extragalactic background light and the history of the universe.

\section{MATERIALS AND METHODS}

A preliminary list of radio sources at high redshift was obtained from the NASA Extragalactic Database (NED). NED is a comprehensive database of extragalactic objects with known positions and redshifts [4]. Two parameters were selected in the search query: radio emission in the $21 \mathrm{~cm}$ band $(1.4 \mathrm{GHz})$ greater than $70 \mathrm{mJy}$, and redshift $z \geq 2.5$. With these parameters, 238 objects where obtained to analyze. To determine that there are not missing objects from the $21 \mathrm{~cm}$ band query, sources with radio emission above $70 \mathrm{mJy}$ at $20 \mathrm{~cm}$ and $6 \mathrm{~cm}$ were also selected. In this way 69 more objects were obtained, for a total of 307 sources for the preliminary list.

Sources with a flat radio spectrum $(\alpha \leq 0.5)$ are desired for the catalog. To determine this kind of spectrum it is necessary to obtain precise measurements of each object's flux in at least two different frequencies and calculate their individual spectral indices. 


\section{Finding Fluxes}

The primary flux measurements of interest were at 1.4GHz. The NRAO/VLA Sky Survey (NVSS) was the main source of this data. NVSS is a survey of the sky using the Very Large Array (VLA) radio telescope at a wavelength of $20 \mathrm{~cm}(1.4 \mathrm{GHz})$ and it covers the northern sky (declination $\delta>-40$ ). Radio flux values at $1.4 \mathrm{GHz}$ frequency were obtained for 291 of the 307 sources in the preliminary list. Two of these objects were determined not to be radio sources.

The remaining 16 fluxes where south of the declination limit in the northern hemisphere surveys, for these 16 we obtained fluxes at $0.843 \mathrm{GHz}$ from the Sydney University Molonglo Sky Survey (SUMSS), a deep radio survey of the entire sky south of $\delta=-30$ degrees, that uses the Molonglo Observatory Synthesis Telescope.

Radio fluxes were also gathered at 4.85, 0.365, and 8.4GHz. Flux measurements at 4.85GHz were found in the 87 Green Bank (87GB) catalog from the VizieR service web page [5] and the Parkes-MIT-NRAO (PMN) surveys. The $87 \mathrm{~GB}$ is a catalog of radio sources covering 6.0 steradians of sky that used the 91m telescope in Green Bank in 1987 and the PMN surveys are source catalogs for equatorial zone. The Texas survey of radio sources provided flux measurements at $0.365 \mathrm{GHz}$. This survey was carried out with the Texas Interferometer during 1974-1983.

We also made use of the radio flux at $8.4 \mathrm{GHz}$, as found by the Cosmic Lens All-Sky Survey (CLASS), to verify spectral index calculations. CLASS is a survey for gravitationally lensed compact radio sources observed with the VLA. Table 1 summarizes the surveys from which detections were obtained.

\section{Finding Spectral indices}

Once the fluxes were obtained the spectral index was calculated between two radio fluxes by using the following formula:

$$
\alpha=\log \left(\frac{F_{2}}{F_{1}}\right) / \log \left(\frac{v_{1}}{v_{2}}\right)
$$


Where $F_{1}$ and $F_{2}$ are the flux values obtained at two different frequencies, and $v_{1}$ and $v_{2}$ are their respective frequencies.

\section{RESULTS}

At least two flux measurements were found for 305 of the 307 objects of the preliminary list. This allowed the calculation of all but two of the spectral indices of all the objects from the preliminary list. All the data obtained for each source was tabulated.

Table 2 displays a sample of the table of the 307 sources with positions, redshifts, flux values at 5 different frequencies, and their calculated spectral index or indices if they had more then two flux values. Spectral indices were calculated for the following flux ratios: $F_{4.85 \mathrm{GHz}} / F_{1.4 \mathrm{Ghz}}$, $F_{4.85 \mathrm{GHz}} / F_{0.843 \mathrm{GHz}}, F_{1.4 \mathrm{GHz}} / F_{0.365 \mathrm{GHz}}$, and $F_{8.4 \mathrm{GHz}} / F_{1.4 \mathrm{GHz}}$.

Figure 1 shows the SED of a well-studied blazar 3C279. Figure 2 and 3 are flux vs. spectral index distribution plots at different frequencies. Of the 305 sources 215 had a spectral index of $\alpha \leq 0.5$. Two additional sources with $\alpha \leq 0.7$ were characterized in the literature as blazars so were also included in the flat-spectrum sample. Figure 4 shows the redshift distribution of the 217 flat-radio spectrum sources from our catalog.

\section{DISCUSSION AND CONCLUSION}

The ultimate goal of this project was to compile a catalog of high-redshift blazars to be found by GLAST. The calculation of the spectral index of each individual object was needed in order to identify blazars from the preliminary list. Because the positions of the sources varied in the sky, it was needed to find as many flux values in different frequencies as possible to have at least two flux measurements for each source to obtain their spectral indices. Only two spectral indices could not be calculated and we succeeded in identifying 217 objects as flat-radio spectrum sources. 


\section{ACKNOWLEDGEMENTS}

I would like to thank my mentor, Jennifer Carson, for her great guidance, dedication, and patience through my learning process. I also thank Teddy Cheung for teaching me the use of databases, surveys, and catalogs from the web and making sure I had all the resources to complete this project. I thank the U.S. Department of Energy, Office of Science for the opportunity I was given to participate in the SULI program and have such an important learning experience.

\section{REFERENCES}

[1] D. J. Thompson "Blazar Variability: A Gamma-Ray perspective" Blazar Variability Workshop II: Entering the GLAST Era ASP Conference Series, Vol. 350, 2006

[2] H. R. Miller, K. Marshall, J. R. Webb, and M. F. Aller "The Capabilities of the GLAST LAT for Studies of BlazarVariability" in ASP Conference Series, Vol. 350, 2006

[3] G.L. Verschuur, K.I. Kellermann. "Galactic and Extragalactic Radio Astronomy" Springer-Verlag second edition 1988.

[4] NASA Extragalactic Database. http://nedwww.ipac.caltech.edu/index.html

[5] VIzieR service webpage. http://vizier.u-strasbg.fr/viz-bin/VizieR 


\begin{tabular}{|l|r|r|}
\hline & & \\
Survey & Frequency $(\mathrm{GHz})$ & Number of Objects \\
\hline NVSS & 1.1 & 291 \\
SUMSS & 0.843 & 30 \\
87GB & 4.85 & 205 \\
PMN & 4.85 & 66 \\
Texas & 0.365 & 183 \\
& & \\
\hline
\end{tabular}

Table 1. Number of objects with detected flux at 4.85, 1.4, 0.843 , and $0.365 \mathrm{GHZ}$

\begin{tabular}{|c|c|c|c|c|c|c|c|c|c|c|c|c|}
\hline Object Name & RA \& DEC Postition: & & 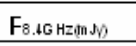 & $\mathrm{F}_{t .85 \mathrm{G} H\left(\ln \Delta_{f}\right)}$ & 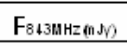 & 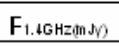 & $\mathrm{F}_{365 \mathrm{MHZ} \text { (n) }}$ & $z$ & $\begin{array}{c}\boldsymbol{\alpha} \\
4.85 \mathrm{GHz} / 1.4 \mathrm{GHz} \\
\end{array}$ & $\begin{array}{c}\underset{\alpha}{\alpha} \\
1.4 \mathrm{GZ} 365 \mathrm{MHz} \\
\end{array}$ & $\begin{array}{c}\boldsymbol{\alpha} \\
8.4 \mathrm{GZ} / .4 \mathrm{G} \mathrm{Hz} \\
\end{array}$ & $\begin{array}{c}\boldsymbol{\alpha} \\
4.25 \mathrm{G} \mathrm{Hz} / 0.843 \mathrm{GHz}\end{array}$ \\
\hline IAG 1 J000 107+1914 & $\infty 018.53+191434.3$ & wVss & 504.2 & 197 & & 265.1 & 221 & 3.1 & 0.24 & -0.14 & -0.36 & \\
\hline [HB89] 0004+171 & $\infty 0547.35+172815.4$ & nVSS & 95.4 & 159 & & 225.5 & 225 & 2.89 & 0.28 & 0.00 & 0.48 & \\
\hline 'MG1 J000655+1416 & $\infty 0557.52+141546.9$ & nvss & 79.6 & 121 & & 183.4 & 381 & 3.2 & 0.33 & 0.54 & 0.47 & \\
\hline Pis $0007+016$ & $\infty 0958.53+01555.2$ & NVSS & 98.2 & 140 & & 270.7 & 325 & 2.95 & 0.53 & 0.14 & 0.57 & \\
\hline PIS $0012-312$ & $\infty 1437.96-305918.8$ & nvss & & 140 & 226.4 & 192.8 & & 2.79 & 0.26 & & & 0.27 \\
\hline [HB89]0014+813 & $\infty 178.51+81358.3$ & nVSS & 1303.8 & & & 692.5 & & 3.37 & & & -0.35 & \\
\hline $87 G 6001510.9+525527$ & $\infty 1751.78+531219.4$ & nVSS & & 650 & & 393.3 & & 2.57 & -0.40 & & & \\
\hline PrS $0017-307$ & $\infty 1942.72-303119.9$ & nvss & & 649 & 504.5 & 507 & 496 & 2.58 & -0.20 & -0.02 & & -0.14 \\
\hline ' $630020+40$ & $\infty 2241.33+4199.1$ & nVSS & & 40 & & 172.9 & 767 & 2.99 & 1.18 & 1.11 & & \\
\hline B R J00305 129 & $\infty 0303436-512946.2$ & sumas & & 76 & 70.4 & & & 4.17 & & & & -0.04 \\
\hline
\end{tabular}

Table 2. Sample table of 307 sources preliminary list. Position, obtained flux measurements, and calculated spectral indices.

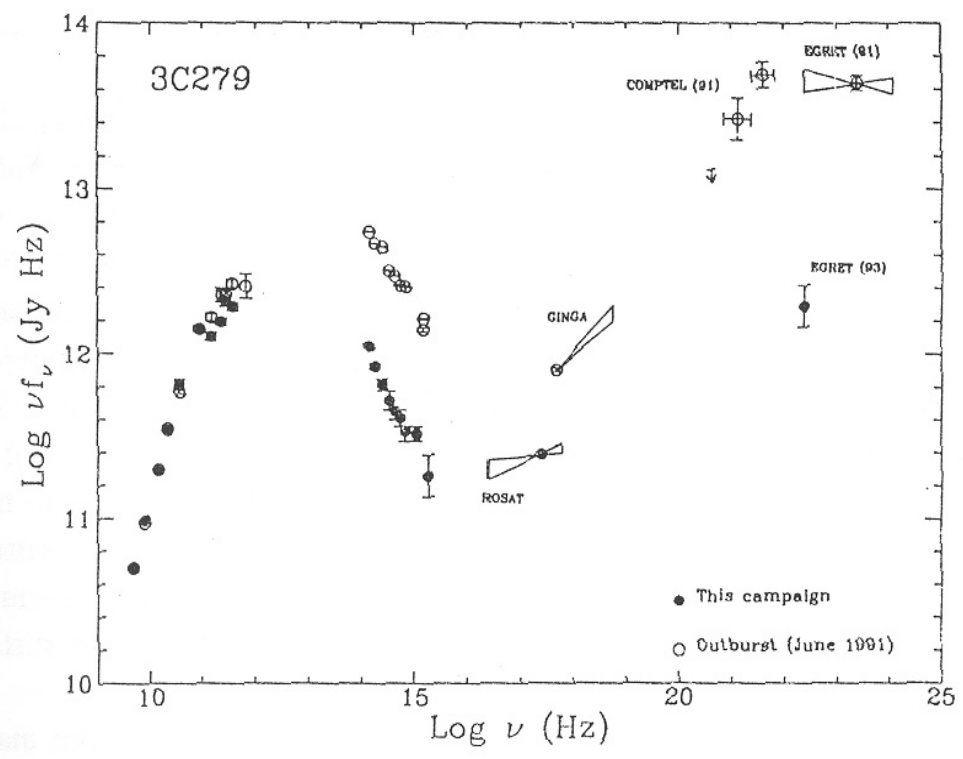

Figure 1. Spectral Energy Distribution of a well-studied blazar 3C279. Double peak signature can be seen from the data points taken at two observational campaigns. Maraschi et al. 1994. 


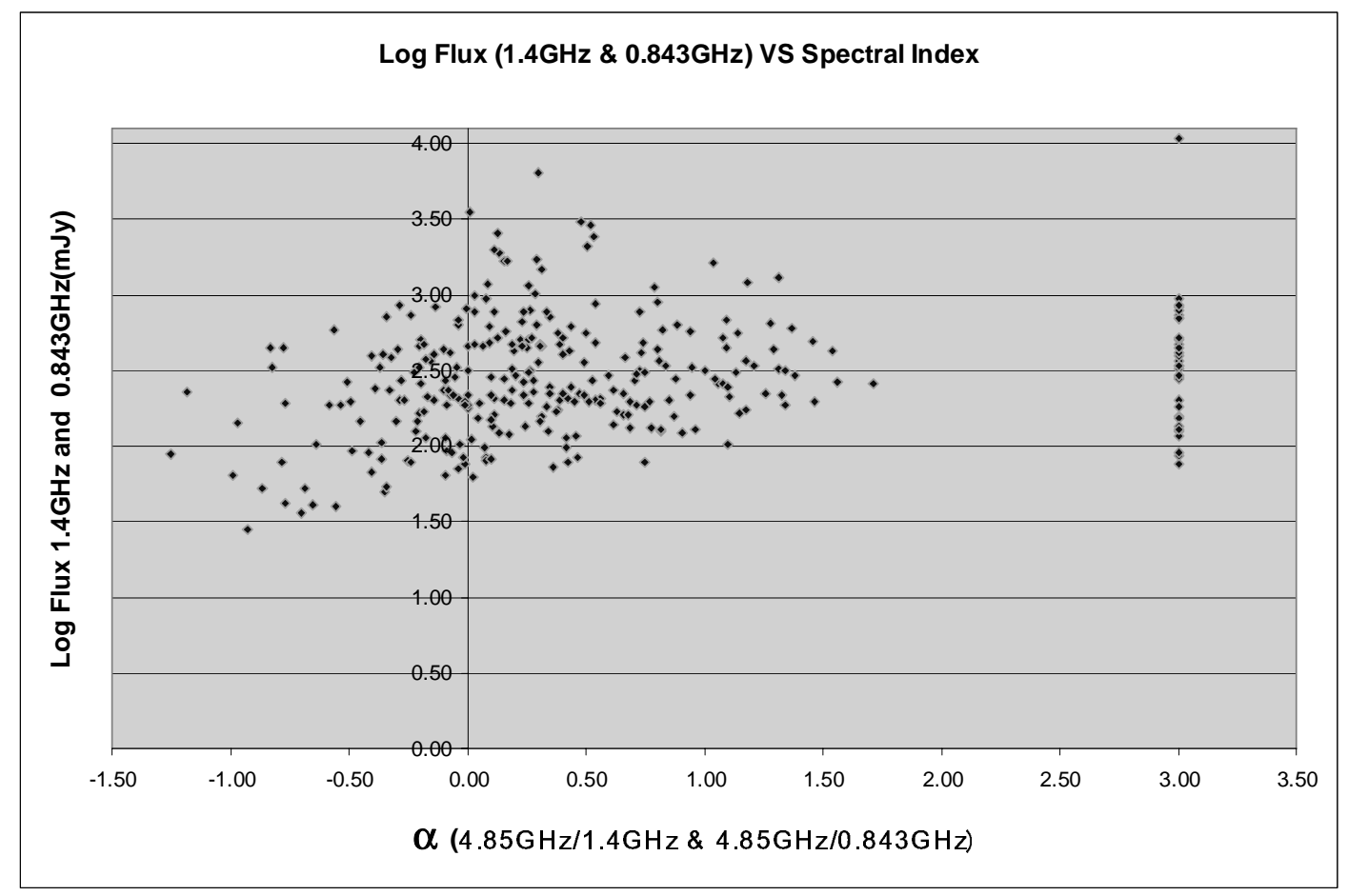

Figure 2. Flux at $1.4 \mathrm{GHz}$ and $0.843 \mathrm{GHz}$ vs. Spectral Index distribution calculated with flux ratio of 4.85GHz/1.4GHz and $4.85 \mathrm{GHz} / 0.843 \mathrm{GHz}$. A value of 3.0 was given to sources with not found spectral index

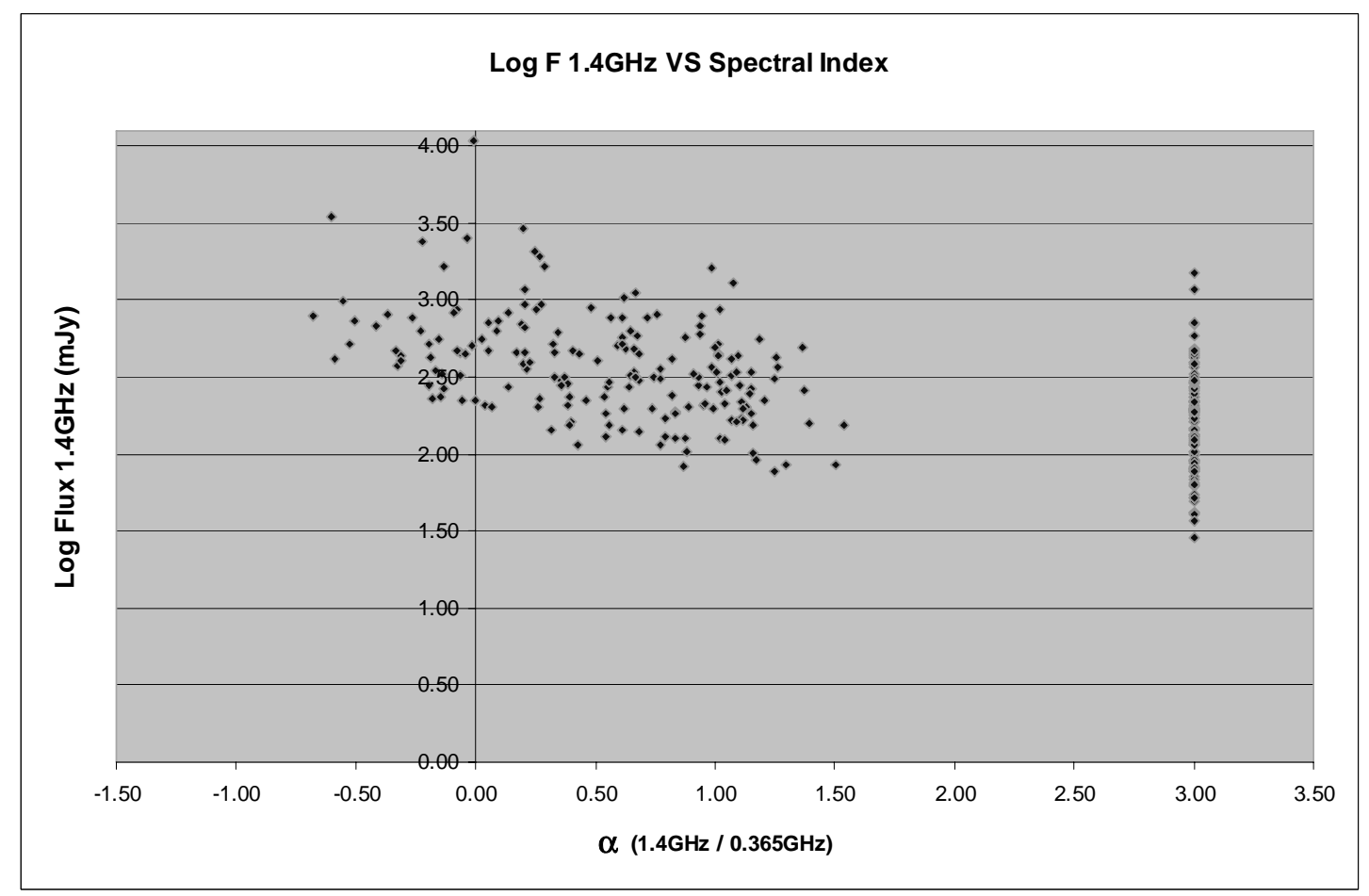

Figure 3 Flux at $1.4 \mathrm{GHz}$ vs. Spectral Index distribution calculated with flux ratio of $1.4 \mathrm{GHz} / 0.365 \mathrm{GHz}$ 


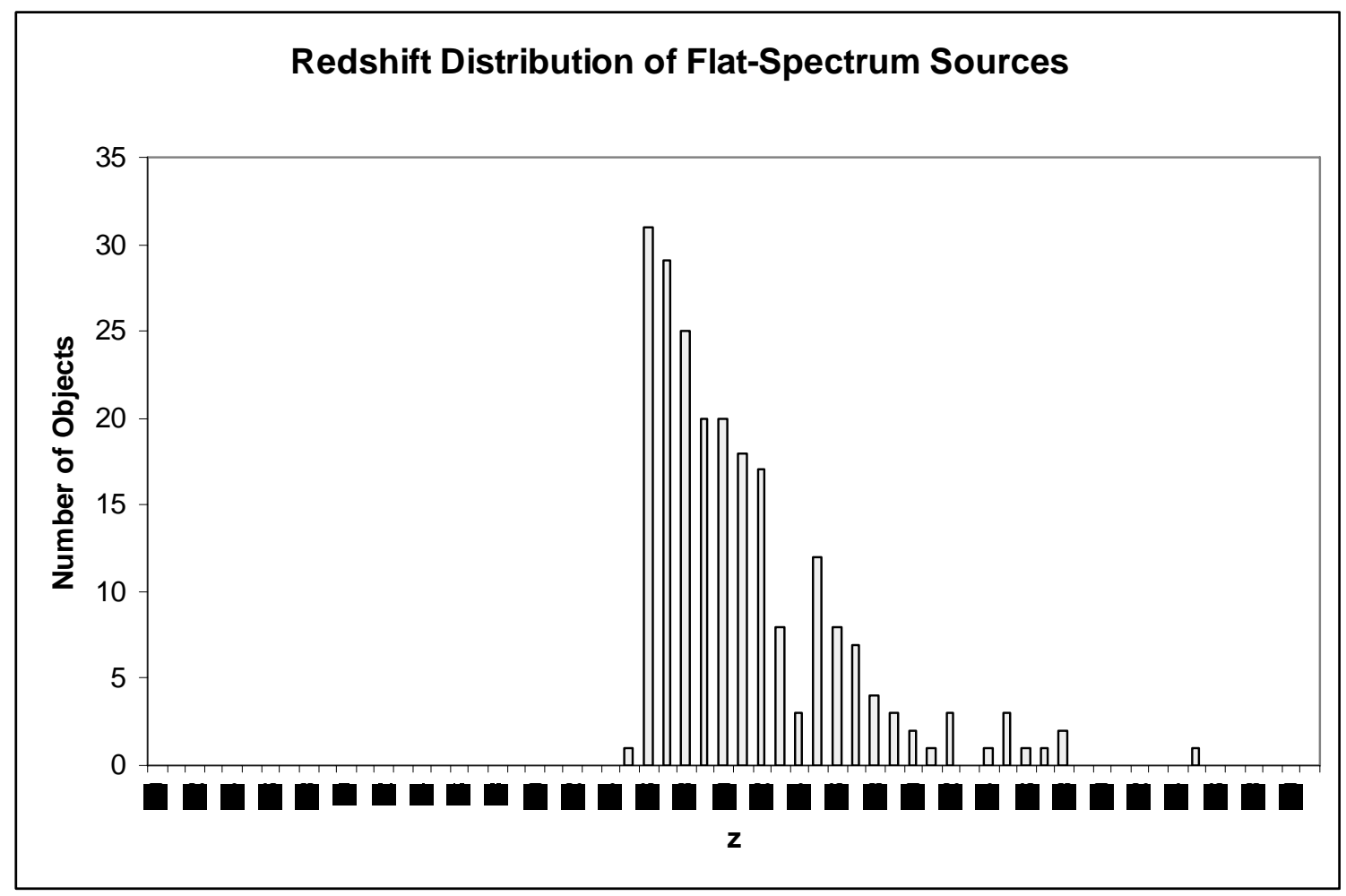

Figure 4. Redshift Distribution of all flat-radio spectrum sources 